\title{
Dynamic Sliding Mode Control Design Based on an Integral Manifold for Nonlinear Uncertain Systems
}

\author{
Qudrat Khan, ${ }^{1}$ Aamer Iqbal Bhatti, ${ }^{2}$ and Antonella Ferrara ${ }^{3}$ \\ ${ }^{1}$ Centre of Advanced Studies in Telecommunications (CAST), COMSATS, Park Road, Chak Shahzad, Islamabad 44000, Pakistan \\ ${ }^{2}$ Department of Electronic Engineering, MAJU, Express Highway, Kahuta Road, Islamabad 44000, Pakistan \\ ${ }^{3}$ Department of Engineering, University of Pavia, Pavia, Italy
}

Correspondence should be addressed to Qudrat Khan; qudratullahqau@gmail.com

Received 27 May 2013; Revised 21 October 2013; Accepted 23 October 2013; Published 2 January 2014

Academic Editor: Huai-Ning Wu

Copyright (C) 2014 Qudrat Khan et al. This is an open access article distributed under the Creative Commons Attribution License, which permits unrestricted use, distribution, and reproduction in any medium, provided the original work is properly cited.

\begin{abstract}
An output feedback sliding mode control law design relying on an integral manifold is proposed in this work. The considered class of nonlinear systems is assumed to be affected by both matched and unmatched uncertainties. The use of the integral sliding manifold allows one to subdivide the control design procedure into two steps. First a linear control component is designed by pole placement and then a discontinuous control component is added so as to cope with the uncertainty presence. In conventional sliding mode the control variable suffers from high frequency oscillations due to the discontinuous control component. However, in the present proposal, the designed control law is applied to the actual system after passing through a chain of integrators. As a consequence, the control input actually fed into the system is continuous, which is a positive feature in terms of chattering attenuation. By applying the proposed controller, the system output is regulated to zero even in the presence of the uncertainties. In the paper, the proposed control law is theoretically analyzed and its performances are demonstrated in simulation.
\end{abstract}

\section{Introduction}

Output feedback sliding mode control techniques proved themselves to be the good candidate for systems where only output is measurable and its derivatives can be estimated accurately. Linear systems or systems which could be easily lineralized are addressed in Edwards and Spurgeon [1]. Nonlinear systems with measurable outputs are for instance dealt with via Dynamic Sliding Mode Control (DSMC) ([24], where the original system is replaced with a differential input-output form often called Fliess Controllable Canonical form or Local generalized controllable canonical (LGCC) form, by using some nonlinear transformation. Asymptotic stabilization of LGCC forms by means of DSMC provided satisfactory results. Traditionally, this control methodology based on the sliding mode control (SMC) theory [5] refers to the case of uncertain systems with matched uncertainties (see, [1] for a definition of this class of uncertainties). However, there are many systems affected by uncertainties which do not satisfy the matching condition. To solve this problem, various methods have been proposed in the literature (see, e.g., [6-11]). These papers of Scararat, Swaroop, and Ferrara relied on a backstepping based SMC design to relax the matching conditions.

Nonlinear systems often do not remain robust against uncertainties even of matched nature in the so-called reaching phase. Therefore, an approach capable of eliminating this phase in the controlled system evolution was proposed in [12]. This approach is based on the design of an integral sliding manifold and is called integral sliding mode control (ISMC). Levant and Alelishvili [13] synthesized higher order sliding mode technique (see, e.g., [14-19]) with integral sliding mode technique to improve the robustness and to alleviate chattering. Choi [20] proposed a linear matrix inequality (LMI) based sliding surface design method for integral sliding mode control of systems with unmatched norm bounded uncertainties. Further, Park et al. [21] extended Choi's method and proposed a dynamical output feedback variable structure control law with high gain to deal with the same problem. Xiang et al. [22] applied an iterative LMI method to avoid the high gain related problems. In this context, da Silva et al. 
[23] developed an algorithm in which the existence and the reachability problems have been formulated using a polytopic description in order to tackle unmatched uncertainties with reduced chattering. Cao and $\mathrm{Xu}$ [24] proposed a nonlinear integral-type sliding surface for the system in the presence of both matched and unmatched uncertainties. The stability of the controlled system with unmatched uncertainties depends on the controlled nominal system and on the nature and size of the equivalent unmatched uncertainties. In the aforementioned approaches, robustness is ensured but with a compromise on chattering alleviation. Castaños and Fridman [25] analyzed the robust features of the integral sliding mode and used $H_{\infty}$ theory to overtake the undesirable effects of the uncertainties. Rubagotti et al. [26] extends the work presented in [25] providing a control law which minimizes the effect of the uncertainties. Chang [27] proposed a dynamic output feedback controller design according to an integral sliding mode approach for linear systems. Note that in the aforementioned papers it is assumed that all the states of the system are available since they are explicitly used to construct the control law.

The main contribution in this work is that the uncertain nonlinear system operating under a class of states dependent matched and unmatched uncertainties is transformed to a generalized controllable canonical form which is analogous to that of $\mathrm{Lu}$ and Spurgeon [4] in some terms. In addition, an integral manifold based control law is developed which establishes sliding mode from the very beginning of the process. The control acting on the original system is obtained as the output of a chain of integrators and is, accordingly, continuous, thus attaining the aim of chattering attenuation. This can be a clear benefit in many applications such as those of mechanical nature, where a discontinuous control action could be nonappropriate. Furthermore, the controller robustness is analyzed in the presence of both matched and unmatched uncertainties. The claim is verified by considering a very simple academic example. Note that the output feedback control of nonlinear systems, which can be put in LGCC form, was previously faced, in a preliminary version, in Khan et al. [28].

The rest of the paper is organized as follows. In Section 2, the problem formulation is presented and in Section 3 the design of the proposed control law is outlined. In Section 4, the stability analysis in the presence of matched and unmatched uncertainties is carried out. A numerical example is discussed in Section 5 which is relevant to a system with relative degree two and is considered to be affected by matched and unmatched uncertainties. Some concluding remarks are reported in Section 6.

\section{Problem Formulation}

Consider a nonlinear SISO dynamic system represented by the state equation analogous to that considered in [24]

$$
\begin{gathered}
\dot{x}=f(x, t)+g(x, t)\left\{u\left(1+\delta_{m}\right)+\Delta g_{m}(x, t)\right\}+f_{u}(x, t), \\
y=h(x),
\end{gathered}
$$

where $x \in R^{n}$ is the state vector, $u \in R$ is scalar control input, $f(x, t)$ and $g(x, t)$ are smooth vector fields, $\delta_{m}$ and $\Delta g_{m}(x, t)$ are matched uncertainties, $f_{u}(x, t)$ is the unmatched uncertainty vector, and $y=h(x) \in R$ is a sufficiently smooth output function. The following assumption is introduced.

Assumption 1. $\delta_{m}, \Delta g_{m}(x, t)$, and $f_{u}(x, t)$ are continuous and bounded with continuous bounded time derivatives for all $(x, t) \in R^{n} \times R_{+}$; that is, $\left|\Delta g_{m}(x, t)\right| \leq \rho_{m},\left|\delta_{m}\right| \leq 1-\varepsilon_{m}$ where $\varepsilon_{m}$ is some positive constant, and $\left\|f_{u}(x, t)\right\| \leq \rho_{u}$.

The problem we want to solve (Problem 1) is that of steering the output $y$ to zero asymptotically; that is, an output regulation problem is considered in the presence of matched and unmatched uncertainties.

In order to design the proposed controller, system (1) is suitably transformed. To this end, we denote with

$$
\begin{gathered}
L_{f} h(x)=\frac{\partial h(x)}{\partial x} f(x)=\nabla h(x) f(x), \\
L_{f_{u}} h(x)=\frac{\partial h(x)}{\partial x} f_{u}=\nabla h(x) f_{u} .
\end{gathered}
$$

Recursively, it can be written as

$$
\begin{gathered}
L_{f}^{0} h(x)=h(x), \\
L_{f}^{j} h(x)=L_{f}\left(L_{f}^{j-1} h(x)\right)=\nabla\left(L_{f}^{j-1} h(x)\right) f(x), \\
L_{f_{u}^{j}}^{j} h(x)=L_{f_{u}}\left(L_{f_{u}}^{j-1} h(x)\right)=\nabla\left(L_{f}^{j-1} h(x)\right) f_{u}, \\
j=1,2, \ldots .
\end{gathered}
$$

The relative degree " $r$ " of the system with respect to the output is the $r$ th derivative of the output function in which the input $u$ appears explicitly [29]. One has

$$
y^{(r)}=L_{f}^{r} h(x)+L_{g}\left(L_{f}^{r-1} h(x)\right) u+\zeta(x, t),
$$

where $\zeta(x, t)$ represents the matched and unmatched uncertainties collection subject to the following conditions:

(1) $L_{g}\left(L_{f}^{i} h(x)\right)=0$ for all $x$ in the neighborhood of $x_{0}$ for $i<r-1$.

(2) $L_{g}\left(L_{f}^{r-1} h(x)\right) \neq 0$.

(3) $L_{g} L_{f_{u}} h(x)=0, L_{g} L_{f_{u}} L_{f} h(x)=0, L_{g} L_{f} L_{f_{u}} h(x)=0$, $L_{g} L_{f_{u}}^{2} h(x)=0$, and so on.

Two possible cases arises for (4).

If $r=n$. then this becomes a trivial case which shows that the system is already in canonical form. This case is neglected in the paper.

If $r<n$, then there exist some positive integer $k$ which satisfy $r+k=n$. 
Then taking the successive $k$ derivatives of (4), one obtains the $n$th derivative of output function as

$$
\begin{aligned}
y^{(n)}= & L_{f}^{n} h(x)+L_{g}\left(L_{f}^{n-1} h(x)\right) u+L_{f} L_{g} L_{f}^{r-1} h(x) u^{(k-1)} \\
& +L_{g}^{2} L_{f}^{r-1} h(x) u u^{(k-1)}+\cdots+L_{g} L_{f}^{r-1} h(x) u^{(k)} \\
& +\zeta^{\star}\left(x, u, \dot{u}, \ldots, u^{(n-1)}, t\right),
\end{aligned}
$$

where $\zeta^{\star}\left(x, u, \dot{u}, \ldots, u^{(n-1)}, t\right)$ is the lumped uncertainty which represents the collection of matched and unmatched uncertainties with their time derivatives.

System (5) can be written as

$$
\begin{aligned}
y^{(n)}= & \varphi(\hat{y}, \widehat{u})+\gamma(\hat{y}) u^{(k)}+\zeta^{\star}\left(x, u, \dot{u}, \ldots, u^{(k-1)}, t\right) \\
= & \varphi(\hat{y}, \widehat{u})+\gamma(\widehat{y})\left\{u^{(k)}\left(1+\delta_{m}\right)+\Delta G_{m}(\widehat{y}, \widehat{u}, t)\right\} \\
& +F_{u}(\widehat{y}, \widehat{u}, t),
\end{aligned}
$$

where

$$
\begin{gathered}
\varphi(\hat{y}, \widehat{u})=L_{f}^{n} h(x)+L_{g}\left(L_{f}^{n-1} h(x)\right) u+\cdots \\
+L_{f} L_{g} L_{f}^{r-1} h(x) u^{(k-1)}+L_{g}^{2} L_{f}^{r-1} h(x) u u^{(k-1)}, \\
\gamma(\widehat{y})=L_{g} L_{f}^{r-1} h(x),
\end{gathered}
$$

$\widehat{y}=\left(y, \dot{y}, \ldots, y^{(n-1)}\right), \widehat{u}=\left(u, \dot{u}, \ldots, u^{(n-1)}\right), \Delta G_{m}(\widehat{y}, \widehat{u}, t)$ is the matched uncertainty term and $F_{u}(\hat{y}, \hat{u}, t)$ is the unmatched uncertainty term.

Note 1. To transform the system (6) into a suitable form, it is first assumed that there are no uncertainties in the system. Therefore, by defining the transformation $y^{(i-1)}=\xi_{i}[3], \widehat{\xi}=$ $\left(\xi_{1}, \xi_{2}, \ldots, \xi_{n}\right)$ for the nominal system, the uncertainties can be represented in the transformed variables like $\zeta_{i}(\widehat{\xi}, \widehat{u}, t)=$ $L_{f_{u}}^{i} h(x)$ for $i=1,2, \ldots, n$ and $\hat{y}=\hat{\xi}$. Finally, system (6) can be written as follows:

$$
\begin{gathered}
\dot{\xi}_{1}=\xi_{2}+\zeta_{1}(\widehat{\xi}, \widehat{u}, t), \\
\dot{\xi}_{2}=\xi_{3}+\zeta_{2}(\widehat{\xi}, \widehat{u}, t), \\
\vdots \\
\dot{\xi}_{n}=\varphi(\widehat{\xi}, \widehat{u})+\gamma(\widehat{\xi})\left\{u^{(k)}\left(1+\delta_{m}\right)+\Delta G_{m}(\widehat{\xi}, \widehat{u}, t)\right\} \\
+F_{u}(\widehat{\xi}, \widehat{u}, t) .
\end{gathered}
$$

The representation in (8) is analogous to the so-called Local generalized controllable canonical (LGCC) form [2], in the sense that it differs from the basic LGCC form since it is also affected by uncertainties. With reference to system (8), the following assumption (which is an alternative form of Assumption 1) is introduced.
Assumption 2. Assume that $|\varphi(\widehat{\xi}, \widehat{u})| \leq C,|\gamma(\widehat{\xi})| \leq K_{M}$, $\left|\Delta G_{m}(\widehat{y}, \widehat{u}, t)\right| \leq \beta_{1},\left|F_{u}(\widehat{\xi}, \widehat{u}, t)\right| \leq \beta_{2}$, and $\left|\zeta_{i}(\widehat{\xi}, \widehat{u}, t)\right| \leq$ $\mu_{i} ; i=1,2, \ldots, n-1$, where $\beta_{1}, \beta_{2}$ and $\mu_{i}$ are positive constants. Furthermore, consider that $\zeta_{1}(\widehat{\xi}, \widehat{u}, t)+\zeta_{2}(\widehat{\xi}, \widehat{u}, t)+$ $\cdots+F_{u}(\widehat{\xi}, \widehat{u}, t) \cong \Delta \Phi(\widehat{\xi}, \widehat{u}, t)$ and $|\Delta \Phi(\widehat{\xi}, \widehat{u}, t)| \leq \tau$.

Now, note that the nominal system corresponding to system (8) is given by

$$
\begin{gathered}
\dot{\xi}_{1}=\xi_{2}, \\
\dot{\xi}_{2}=\xi_{3}, \\
\vdots \\
\dot{\xi}_{n}=\varphi(\widehat{\xi}, \widehat{u})+\gamma(\widehat{\xi}) u^{(k)}=\psi\left(\widehat{\xi}, \widehat{u}, u^{(k)}\right) .
\end{gathered}
$$

Definition 3. The differential input output (I-O) form (or LGCC form) is termed as proper if [4]

(1) it is single input single output;

(2) $\psi\left(\widehat{\xi}, \widehat{u}, u^{(k)}\right) \in C^{1}$;

(3) the following regularity condition is satisfied:

$$
\operatorname{det}\left[\frac{\partial \psi\left(\widehat{\xi}, \widehat{u}, u^{(k)}\right)}{\partial u^{(k)}}\right] \neq 0 .
$$

Definition 4. The zero dynamics of the system in (9) are defined as [4]

$$
\psi\left(0, \widehat{u}, u^{(k)}\right)=0 .
$$

The system in (9) is called minimum phase if the zero dynamics defined in (11) are uniformly asymptotically stable. Note that the zero dynamics in the I-O form are the dynamics of the control and is the generalization of the definition in Fliess [30]. They are different from the zero dynamics mentioned in Isidori [29] which are the dynamics of the uncontrollable states.

Assumption 5. System (9) is proper and minimum phase according to Definitions 3 and 4, respectively.

Now the original control problem (Problem 1) can be reformulated with reference to system (8) under Assumption 2 and to the nominal system in (9) subject to Assumption 5. The new problem (Problem 2) is that of steering the state vector $\xi=\left[\xi_{1}, \xi_{2}, \ldots, \xi_{n}\right]^{T}$ of system (8) to zero asymptotically inspite of the presence of matched and unmatched uncertainties; that is, a state regulation problem is now considered. Clearly the solution to Problem 2 implies the solution to Problem 1, since $\xi_{1}=y=h(x)$.

\section{The Proposed Control Law Design}

In analogy with Khan et al. [31], where only the presence of matched uncertainties was considered, we propose a control law of dynamic nature which can be expressed as

$$
u^{(k)}=u_{0}^{(k)}+u_{1}^{(k)} .
$$


The first part $u_{0}^{(k)} \in R$ is continuous and stabilizes the system at the equilibrium point, while the second part $u_{1}^{(k)} \in R$ is discontinuous in nature and can be classified as an integral $\mathrm{SMC}$. Its role is to reject uncertainties. In the next subsections, the design of $u_{0}^{(k)} \in R$ and $u_{1}^{(k)} \in R$ will be discussed. Starting from the nominal case and then moving to the case in which the presence of matched and unmatched uncertainties are also considered.

\subsection{The Nominal Case}

3.1.1. Design of $u_{0}^{(k)}$. The nominal system in (9) in alternative form can be written as

$$
\begin{gathered}
\dot{\xi}_{1}=\xi_{2}, \\
\dot{\xi}_{2}=\xi_{3}, \\
\vdots \\
\dot{\xi}_{n}=\chi\left(\widehat{\xi}, \widehat{u}, u^{(k)}\right)+u^{(k)},
\end{gathered}
$$

where

$$
\chi\left(\widehat{\xi}, \widehat{u}, u^{(k)}\right)=\varphi(\xi, \widehat{u})+(\gamma(\widehat{\xi})-1) u^{(k)}
$$

In the design of $u_{0}^{(k)}$, system (13) is first considered to be independent of nonlinearities; that is, $\chi\left(\widehat{\xi}, \widehat{u}, u^{(k)}\right)=0$, and it is also supposed to operate under $u_{0}^{(k)}$ only. Then, system (13) becomes

$$
\begin{gathered}
\dot{\xi}_{1}=\xi_{2}, \\
\dot{\xi}_{2}=\xi_{3}, \\
\vdots \\
\dot{\xi}_{n}=u_{0}^{(k)} .
\end{gathered}
$$

This is a linear system, so it can be written as

$$
\dot{\xi}=A \xi+B u_{0}^{(k)},
$$

where

$$
\begin{gathered}
A=\left[\begin{array}{cc}
0_{(n-1) \times 1} & I_{(n-1)(n-1)} \\
0_{1 \times 1} & 0_{1 \times(n-1)}
\end{array}\right], \\
B=\left[\begin{array}{c}
0_{(n-1) \times 1} \\
1
\end{array}\right] .
\end{gathered}
$$

For the sake of simplicity, the input $u_{0}^{(k)}$ is designed via pole placement; that is,

$$
u_{0}^{(k)}=-K_{0}^{T} \xi
$$

3.1.2. Design of $u_{1}^{(k)}$. Now in order to achieve the desired performance, robust compensation of the uncertainties is needed. To this end we select the following sliding manifold of integral type [12]:

$$
\sigma(\xi)=\sigma_{0}(\xi)+z,
$$

where $\sigma_{0}(\xi)$ is a conventional sliding surface which is mathematically defined by $\sigma_{0}(\xi)=\sum_{i=1}^{n} c_{i} \xi_{i}$, with $c_{n}=1$, and $z$ is the integral term. The time derivative of (19) along (9) yields

$$
\dot{\sigma}(\xi)=\sum_{i=1}^{n-1} c_{i} \xi_{i+1}+\chi\left(\widehat{\xi}, \widehat{u}, u^{(k)}\right)+u_{0}^{(k)}+u_{1}^{(k)}+\dot{z} .
$$

Now, choose $\dot{z}$ with the following expression:

$$
\begin{gathered}
\dot{z}=-\left(\sum_{i=1}^{n-1} c_{i} \xi_{i+1}+u_{0}^{(k)}\right), \\
z(0)=-\sigma_{0}(\xi(0)) .
\end{gathered}
$$

Then, (20) becomes

$$
\begin{aligned}
\dot{\sigma}(\xi) & =\chi\left(\widehat{\xi}, \widehat{u}, u^{(k)}\right)+u_{1}^{(k)} \\
& =\varphi(\widehat{\xi}, \widehat{u})+(\gamma(\widehat{\xi})-1) u_{0}^{(k)}+\gamma(\widehat{\xi}) u_{1}^{k} .
\end{aligned}
$$

This initial condition $z(0)$ is adjusted in such a way to meet the requirement $\sigma(0)=0$.

Taking into account the reachability condition defined as follows [5]:

$$
\dot{\sigma}=-K \operatorname{sign} \sigma
$$

and comparing (22) with (23), the expression of the discontinuous control component $u_{1}^{(k)}$ becomes

$$
u_{1}^{(k)}=\frac{1}{\gamma(\widehat{\xi})}\left(-\varphi(\widehat{\xi}, \widehat{u})-(\gamma(\widehat{\xi})-1) u_{0}^{(k)}-K \operatorname{sign} \sigma\right) .
$$

This control law enforces sliding mode along the sliding manifold defined in (19). The constant $K$ can be selected according to the subsequent stability analysis.

Thus, the final control law becomes

$$
u^{(k)}=-K_{0}^{T} \xi+\frac{1}{\gamma(\widehat{\xi})}\left(-\varphi(\widehat{\xi}, \widehat{u})-(\gamma(\widehat{\xi})-1) u_{0}^{(k)}-K \operatorname{sign} \sigma\right) .
$$

Note that this control law can be implemented by integrating the derivative of the control, $u^{(k)}$, " $k$ " times so that the control input actually applied to the system is continuous. This can be a benefit for various class of systems such as those of mechanical type, for which a discontinuous control action could be disruptive.

Remark 6. The coefficients of the conventional sliding surface are chosen by tacking into the dynamic response of the system. However, in real applications these constants can also be optimized using LMIs methods.

Remark 7. The proposed methodology needs the availability of the system output and of its derivatives for the controller implementation. In case the output derivatives are not available for measurements, one can use for instance a finite time sliding mode differentiator like the one proposed in [16] to reconstruct them. 


\section{Stability Analysis}

In this section, the proposed control law when applied to the uncertain nonlinear system in question is theoretically analyzed. First the case in which only matched uncertainties are present will be discussed, and then the more general case of matched and unmatched uncertainties will be considered.

4.1. The System Operating under Matched Uncertainties. Now we assume that the system operates only under matched uncertainties. Thus, system (8) with matched uncertainties becomes

$$
\begin{gathered}
\dot{\xi}_{1}=\xi_{2}, \\
\dot{\xi}_{2}=\xi_{3}, \\
\vdots \\
\dot{\xi}_{n}=\varphi(\widehat{\xi}, \widehat{u})+\gamma(\widehat{\xi})\left\{u^{(k)}\left(1+\delta_{m}\right)+\Delta G_{m}(\widehat{\xi}, \widehat{u}, t)\right\} .
\end{gathered}
$$

To show that this system is stabilized in finite time in the presence of matched uncertainties, the following theorem can be stated.

Theorem 8. Consider that Assumptions 2 and 5 are satisfied. The sliding surface is chosen as $\sigma(\xi)=0$, where $\sigma(\xi)$ is defined in (19), and the control law $u^{(k)}$ is selected according to (25). If the gain $K$ is chosen according to the following condition:

$$
K \geq \frac{1}{\left(2-\varepsilon_{m}\right)}\left[\left(1-\varepsilon_{m}\right)\left|u_{0}^{(k)}\right|+\left(1-\varepsilon_{m}\right) C+K_{M} \beta_{1}+\eta_{1}\right],
$$

where $\eta_{1}$ is a positive constant, then the finite time enforcement of a sliding mode on $\sigma(\xi)=0$ is guaranteed in the presence of matched uncertainties.

Proof. To prove that the sliding mode can be enforced in finite time, differentiating (19) along the dynamics of (26) and then substituting (25), one has

$$
\begin{aligned}
\dot{\sigma}(\xi)= & \sum_{i=1}^{n-1} c_{i} \xi_{i+1}+u_{0}^{(k)}-K \operatorname{sign} \sigma \\
& +\delta_{m}\left[-\varphi(\widehat{\xi}, \widehat{u})+u_{0}^{(k)}-K \operatorname{sign} \sigma\right] \\
& +\gamma(\widehat{\xi}) \Delta G_{m}(\widehat{\xi}, \widehat{u}, t)+\dot{z} .
\end{aligned}
$$

Substituting (21) into (28) and then rearranging, one obtains

$$
\begin{aligned}
\dot{\sigma}(\xi)= & -K \operatorname{sign} \sigma+\delta_{m}\left[-\varphi(\widehat{\xi}, \widehat{u})+u_{0}^{(k)}-K \operatorname{sign} \sigma\right] \\
& +\gamma(\widehat{\xi}) \Delta G_{m}(\widehat{\xi}, \widehat{u}, t) .
\end{aligned}
$$

Now, by considering as a Lyapunov candidate function $V=$ $(1 / 2) \sigma^{2}$, the time derivative of this function becomes

$$
\dot{V}=\sigma \dot{\sigma}
$$

So, by using (29) in (30), one has

$$
\begin{aligned}
\dot{V} \leq-|\sigma|[ & -K\left(1+\left|\delta_{m}\right|\right)+\left|\delta_{m}\right||\varphi(\widehat{\xi}, \widehat{u})| \\
& \left.+\left|\delta_{m}\right|\left|u_{0}^{(k)}\right|+\left|\gamma(\widehat{\xi}) \Delta G_{m}(\widehat{\xi}, \widehat{u}, t)\right|\right] .
\end{aligned}
$$

In view of Assumption 2, the above expression can be written as

$$
\begin{gathered}
\dot{V} \leq|\sigma|\left[\left(1-\varepsilon_{m}\right)\left|u_{0}^{(k)}\right|-\left(2-\varepsilon_{m}\right) K+\left(1-\varepsilon_{m}\right) C+K_{M} \beta_{1}\right] \\
\dot{V} \leq-|\sigma| \eta_{1}<0,
\end{gathered}
$$

provided that

$$
K \geq \frac{1}{\left(2-\varepsilon_{m}\right)}\left[\left(1-\varepsilon_{m}\right)\left|u_{0}^{(k)}\right|+\left(1-\varepsilon_{m}\right) C+K_{M} \beta_{1}+\eta_{1}\right]
$$

as in (27). Note that (32) can also be written as

$$
\dot{V}+\sqrt{2} \eta_{1} \sqrt{V}<0 .
$$

This implies that $\sigma(\xi)$ converges to zero in a finite time $t_{s}[1]$ such that

$$
t_{s} \leq \sqrt{2} \eta_{1}^{-1} \sqrt{V}(\sigma(0)),
$$

which completes the proof.

Corollary 9. The dynamics of the system (26), with control law (25) and sliding manifold $\sigma(\xi)=0$, with $\sigma(\xi)$ defined in (19), in sliding mode are governed by the linear control law (18).

Proof. The nonlinear system (26) can be written in the following alternate form:

$$
\begin{aligned}
& \dot{\xi}_{1}=\xi_{2}, \\
& \dot{\xi}_{2}=\xi_{3},
\end{aligned}
$$

$$
\begin{aligned}
\dot{\xi}_{n}= & \varphi(\xi, \widehat{u})+(\gamma(\widehat{\xi})-1) u^{(k)}+u_{0}^{(k)} \\
& +u_{1}^{(k)}+\gamma(\widehat{\xi})\left\{u^{(k)} \delta_{m}+\Delta G_{m}(\widehat{\xi}, \widehat{u}, t)\right\} .
\end{aligned}
$$

The time derivative of (19) along (36) yields

$$
\begin{aligned}
\dot{\sigma}(\xi)= & \sum_{i=1}^{n-1} c_{i} \xi_{i+1}+\varphi(\xi, \widehat{u})+(\gamma(\widehat{\xi})-1) u^{(k)} \\
& +u_{0}^{(k)}+u_{1}^{(k)}+\gamma(\widehat{\xi})\left\{u^{(k)} \delta_{m}+\Delta G_{m}(\widehat{\xi}, \widehat{u}, t)\right\}+\dot{z}
\end{aligned}
$$

Substituting (21) into (37), posing $\dot{\sigma}(\xi)=0$, and solving with respect to the control variable $u^{(k)}$, one obtains the so-called equivalent control [5] as

$$
u_{\mathrm{eq}}^{(k)}=-\frac{1}{\gamma(\widehat{\xi})\left(1+\delta_{m}\right)}\left[\varphi(\widehat{\xi}, \widehat{u})-u_{0}^{(k)}+\gamma(\widehat{\xi}) \Delta G_{m}(\widehat{\xi}, \widehat{u}, t)\right] .
$$


Now, using (38) into (36), one has

$$
\dot{\xi}_{s}=A \xi_{s}+B u_{0}^{(k)}
$$

where $\xi_{s}$ is the state of system (26) while in sliding mode. Thus, it is proved that the system in sliding mode operates under the continuous control law and the eigenvalues of the controlled transformed system in sliding mode are those of $A-B K_{0}^{T}$.

4.2. The System Operating under Both Matched and Unmatched Uncertainties. In this subsection, it is now assumed that the considered system operates under both matched and unmatched uncertainties and the control objective is to regulate the output of the system in the presence of these uncertainties. To prove that the proposed control law is capable of compensating for these uncertain terms, the following theorem can be stated.

Theorem 10. Consider that Assumptions 2 and 5 are satisfied. The sliding surface is chosen as $\sigma(\xi)=0$, where $\sigma(\xi)$ is defined in (19), and the control law $u^{(k)}$ is selected according to (25). If the gain $K$ is chosen according to the following condition:

$$
K \geq \frac{1}{\left(2-\varepsilon_{m}\right)}\left[\left(1-\varepsilon_{m}\right)\left|u_{0}^{(k)}\right|+\left(1-\varepsilon_{m}\right) C+K_{M} \beta_{1}+\tau+\eta_{2}\right],
$$

where $\eta_{2}$ is some positive constant, then the finite time enforcement of a sliding mode on $\sigma(\xi)=0$ is guaranteed in the presence of both matched and unmatched uncertainties.

Proof. Considering the time derivative of (19) along (8), and then substituting (25), one has

$$
\begin{aligned}
\dot{\sigma}(\xi)= & \sum_{i=1}^{n-1} c_{i} \xi_{i+1}+u_{0}^{(k)}-K \operatorname{sign} \sigma \\
& +\delta_{m}\left[-\varphi(\widehat{\xi}, \widehat{u})+u_{0}^{(k)}-K \operatorname{sign} \sigma\right] \\
& +\gamma(\widehat{\xi}) \Delta G_{m}(\widehat{\xi}, \widehat{u}, t)+\Delta \Phi(\widehat{\xi}, \widehat{u}, t)+\dot{z}
\end{aligned}
$$

Using (21) in (41), it yields

$$
\begin{aligned}
\dot{\sigma}(\xi)= & -K \operatorname{sign} \sigma+\delta_{m}\left[-\varphi(\widehat{\xi}, \widehat{u})+u_{0}^{(k)}-K \operatorname{sign} \sigma\right] \\
& +\gamma(\widehat{\xi}) \Delta G_{m}(\widehat{\xi}, \widehat{u}, t)+\Delta \Phi(\widehat{\xi}, \widehat{u}, t) .
\end{aligned}
$$

Now, by considering as a Lyapunov candidate function $V=$ $(1 / 2) \sigma^{2}$, the time derivative of this function becomes

$$
\dot{V}=\sigma \dot{\sigma}
$$

Using (42) in (43), one has

$$
\begin{array}{r}
\dot{V} \leq-|\sigma|[- \\
+\left|\gamma\left(1+\left|\delta_{m}\right|\right)+\right| \delta_{m}|| \varphi(\widehat{\xi}) \Delta G_{m}(\widehat{\xi}, \widehat{u}, t)|+| \delta_{m}|| u_{0}^{(k)} \mid \\
+|\Delta \Phi(\widehat{\xi}, \widehat{u}, t)| .
\end{array}
$$

In view of Assumption 2, the above expression can be written as

$$
\begin{gathered}
\dot{V} \leq|\sigma|\left[\left(1-\varepsilon_{m}\right)\left|u_{0}^{(k)}\right|-\left(2-\varepsilon_{m}\right) K+\left(1-\varepsilon_{m}\right) C+K_{M} \beta_{1}+\tau\right] \\
\dot{V} \leq-|\sigma| \eta_{2}<0,
\end{gathered}
$$

provided that

$$
K \geq \frac{1}{\left(2-\varepsilon_{m}\right)}\left[\left(1-\varepsilon_{m}\right)\left|u_{0}^{(k)}\right|+\left(1-\varepsilon_{m}\right) C+K_{M} \beta_{1}+\eta_{2}+\tau\right] .
$$

The expression in (45) can be placed in the same format like that of (34). Note that the finite time $t_{s}$ in this case is given by the formula in (35) with $\eta_{2}$ instead of $\eta_{1}$. Thus it is confirmed that, when the gain of the control law (25) is selected according to (40), the finite time enforcement of the sliding mode is guaranteed in the presence of matched and unmatched uncertainties, which proves the theorem.

Corollary 11. The dynamics of system (8), with control law (25) and an integral manifold $\sigma(\xi)=0$, with $\sigma(\xi)$ defined in (19), in sliding mode are governed by the linear control law (18).

Proof. The proof can be performed by following the same procedure as in the proof of Corollary 9, with the only difference that in this case the equivalent control is equal to

$$
\begin{aligned}
u_{\mathrm{eq}}^{(k)}= & -\frac{1}{\gamma(\widehat{\xi})\left(1+\delta_{m}\right)} \\
& \times\left[\varphi(\widehat{\xi}, \widehat{u})-u_{0}^{(k)}+\gamma(\widehat{\xi}) \Delta G_{m}(\widehat{\xi}, \widehat{u}, t)+\Delta \Phi(\widehat{\xi}, \widehat{u}, t)\right] .
\end{aligned}
$$

\section{Illustrative Example (System with Relative Degree 2)}

Consider the following uncertain nonlinear system [4]:

$$
\begin{gathered}
\dot{x}_{1}=x_{2}+f_{1}(x, t), \\
\dot{x}_{2}=x_{1}^{2}+\left(x_{2}^{2}+1\right) u\left\{\left(1+\delta_{m}\right)+\Delta g_{m}(x, t)\right\}+x_{3}+f_{2}(x, t), \\
\dot{x}_{3}=-x_{3}+x_{2} x_{3}^{2}+f_{3}(x, t),
\end{gathered}
$$

where $x_{1}, x_{2}$, and $x_{3}$ are the states of the nonlinear system. The terms $\delta_{m}, \Delta g_{m}(x, t)$ are matched uncertainties and $f_{i}(x, t)$ are components of the mismatched uncertainty which satisfy Assumptions 1 and 2 and these terms contribute to the system uncertainty with the following mathematical expressions:

$$
\begin{aligned}
f_{1}(x, t)= & -x_{3}+x_{2} x_{3}^{2}+\left(-x_{3}+x_{2} x_{3}^{2}\right)^{2} \\
& +0.25 \sin (t) \cos \left(3 x_{2}\right)+0.26, \\
f_{2}(x, t)= & 0.25 \sin (t) \cos \left(3 x_{2}\right)+0.1,
\end{aligned}
$$



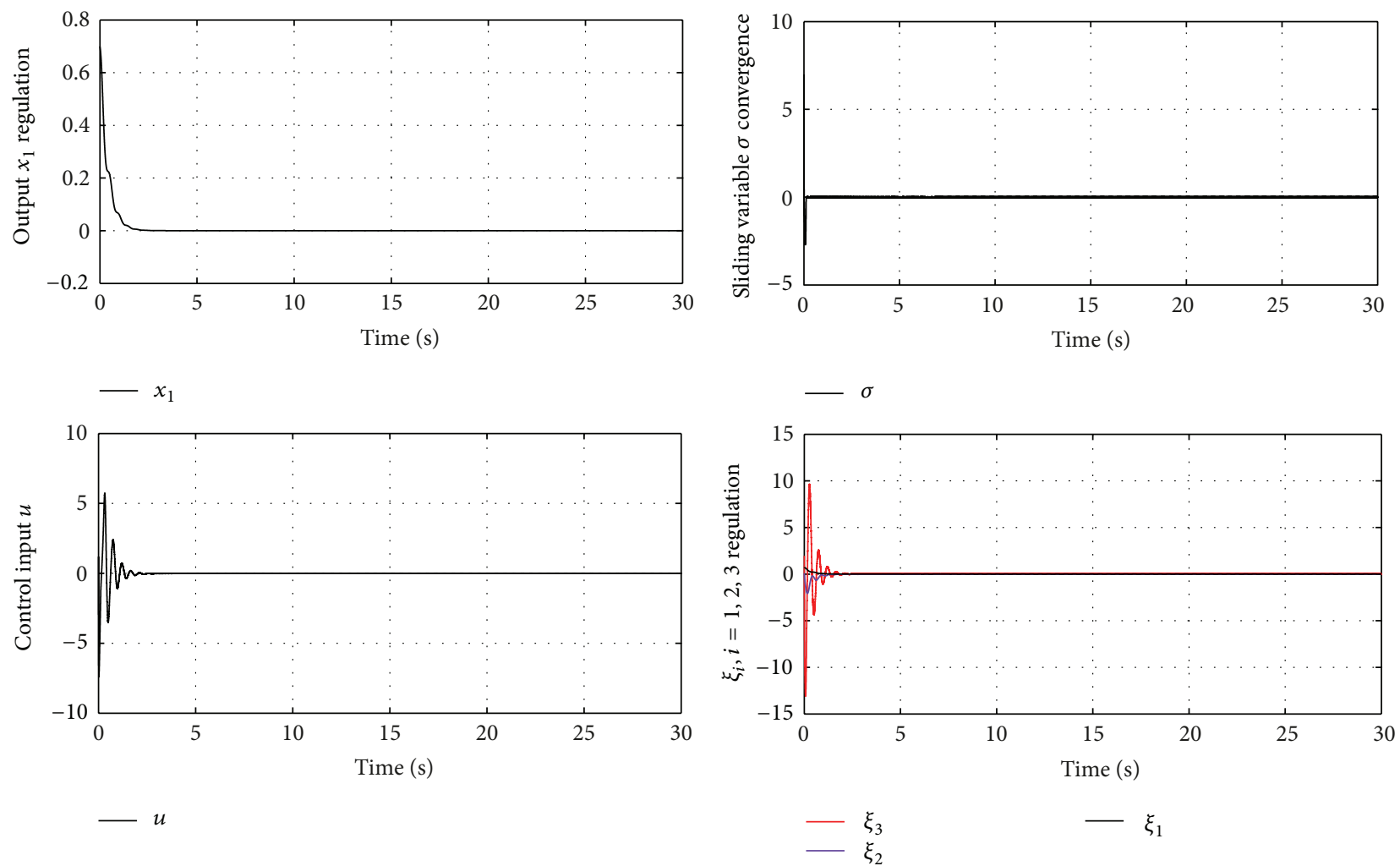

FIGURE 1: Output regulation, control effort, sliding variable convergence and $\left[\xi_{1}, \xi_{2}, \xi_{3}\right]^{T}$ regulation in the presence of matched uncertainty via the proposed control law.

$$
\begin{gathered}
f_{3}(x, t)=-x_{3}+x_{2} x_{3}^{2}+3\left(-x_{3}+x_{2} x_{3}^{2}\right)^{2} \\
+0.25 \sin (t) \cos \left(3 x_{2}\right)+0.1 \\
\Delta g_{m}(x, t)=3\left(-x_{3}+x_{2} x_{3}^{2}\right) \\
\delta_{m}=0.3 \cos \left(\pi t x_{2}\right) .
\end{gathered}
$$

The output of interest is the state variable $x_{1}$. The relative degree of which is 2 . Consequently, the system in (48) can be expressed in LGCC form as follows:

$$
\begin{gathered}
\dot{\xi}_{1}=\xi_{2}+\zeta_{1}(\widehat{\xi}, \widehat{u}, t), \\
\dot{\xi}_{2}=\xi_{3}+\zeta_{2}(\widehat{\xi}, \widehat{u}, t), \\
\dot{\xi}_{3}=\varphi(\widehat{\xi}, \widehat{u})+\gamma(\widehat{\xi}) \dot{u}+\Delta G_{m}(\widehat{\xi}, \widehat{u}, t)+F_{u}(\widehat{\xi}, \widehat{u}, \dot{u}, t),
\end{gathered}
$$

where $(y, \dot{y}, \ddot{y})=\left(\xi_{1}, \xi_{2}, \xi_{3}\right)=\widehat{\xi}, \widehat{u}=u$,

$$
\begin{gathered}
\xi_{1}=x_{1}, \\
\xi_{2}=x_{2}, \\
\xi_{3}=x_{1}^{2}+\left(x_{2}^{2}+1\right) u+x_{3},
\end{gathered}
$$

$$
\begin{gathered}
x_{1}=\xi_{1}, \\
x_{2}=\xi_{2}, \\
x_{3}=\xi_{3}-\xi_{1}^{2}-\left(\xi_{2}^{2}+1\right) u .
\end{gathered}
$$

Thus, one has

$$
\begin{gathered}
\gamma(\widehat{\xi})=\left(\xi_{2}^{2}+1\right), \\
\varphi(\widehat{\xi}, \widehat{u})=2 \xi_{1} \xi_{2}+2 \xi_{2} \xi_{3} u-\left(\xi_{3}-\xi_{1}^{2}-\left(\xi_{2}^{2}+1\right) u\right) \\
+\xi_{2}\left(\xi_{3}-\xi_{1}^{2}-\left(\xi_{2}^{2}+1\right) u\right) .
\end{gathered}
$$

The regularity condition mentioned in Definition 3 holds and the zero dynamics of this system express according to Definition 4 becomes

$$
\dot{u}+u=0 \text {. }
$$

This confirms that the nominal system is minimum phase. The corresponding linear system becomes

$$
\dot{\xi}=A \xi+B \dot{u}_{0}
$$

where

$$
A=\left[\begin{array}{lll}
0 & 1 & 0 \\
0 & 0 & 1 \\
0 & 0 & 0
\end{array}\right], \quad B=\left[\begin{array}{l}
0 \\
0 \\
1
\end{array}\right],
$$



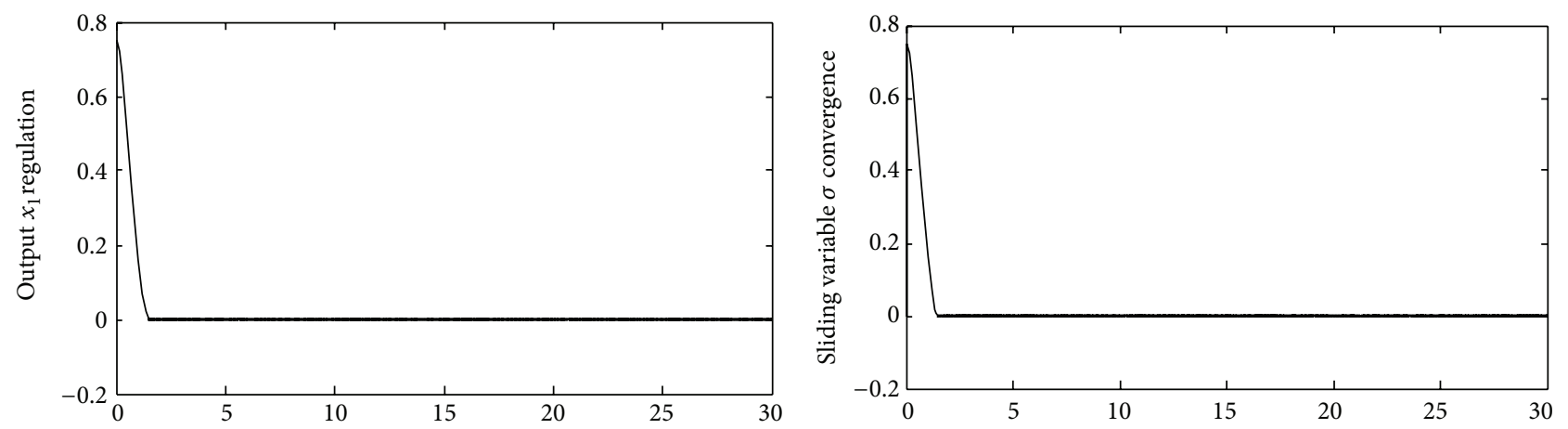

$-x_{1}$
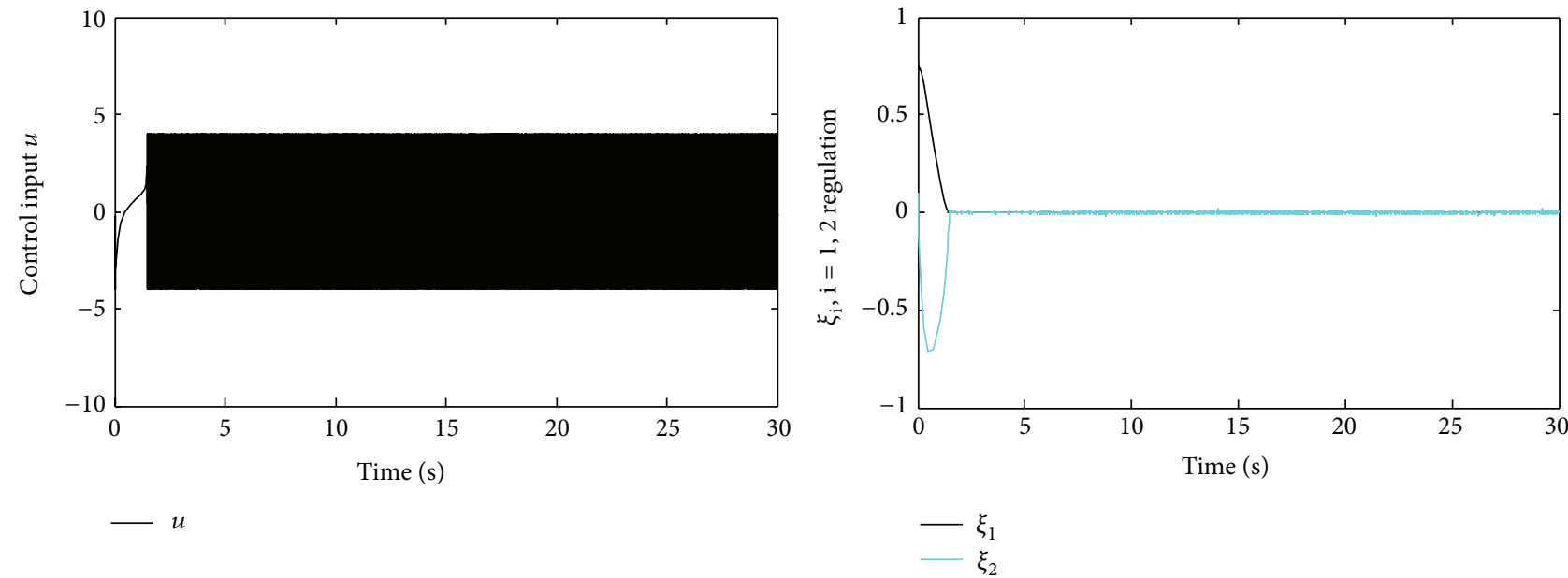

FIGURE 2: Output regulation, control effort, sliding variable convergence, and $\left[\xi_{1}, \xi_{2}\right]^{T}$ regulation in the presence of matched uncertainty via the 2-QCSMC.

and the control law

$$
\dot{u}_{0}=-k_{1} \xi_{1}-k_{2} \xi_{2}-k_{3} \xi_{3}
$$

is obtained by pole assignment. The sliding variable now adopted is

$$
\sigma=c_{1} \xi_{1}+c_{2} \xi_{2}+\xi_{3}+z
$$

with

$$
\begin{gathered}
\dot{z}=-\dot{u}_{0}-\left(c_{1} \xi_{2}+c_{2} \xi_{3}\right), \\
z(0)=0,
\end{gathered}
$$

and the final expression of the control law $\dot{u}$ takes the form

$$
\dot{u}=\dot{u}_{0}+\frac{1}{\gamma(\widehat{\xi})}\left[-\varphi(\widehat{\xi}, \widehat{u})-(\gamma(\widehat{\xi})-1) \dot{u}_{0}-K \operatorname{sign}(\sigma)\right] .
$$

In this study we compare the results of the proposed control law with that of quasicontinuous high order sliding mode controller proposed by Levant in [18]. To apply such an approach, we denote

$$
\begin{aligned}
& s=x_{1}, \\
& \dot{s}=x_{2} .
\end{aligned}
$$

So that the expression of the quasicontinuous sliding mode controller in case of relative degree (2-QCSMC) takes the following form:

$$
u=-\frac{\alpha\left(\dot{s}+|s|^{1 / 2} \operatorname{sign}(s)\right)}{|\dot{s}|+|s|^{1 / 2}},
$$

where $\alpha$ is the controller gain which can be selected according to Bartolini et al. [32]. As proved in Levant [18], the control law (61) provides a finite time sliding mode of the system with a control law which is continuous everywhere except on the second order sliding manifold $s=\dot{s}=0$.

Note 2. It is not necessary that every system whose output is available can be put in the form appearing in (8) and (9).

Case 1 (system operated with matched uncertainty). In this study, the system with matched uncertainties (i.e., with 

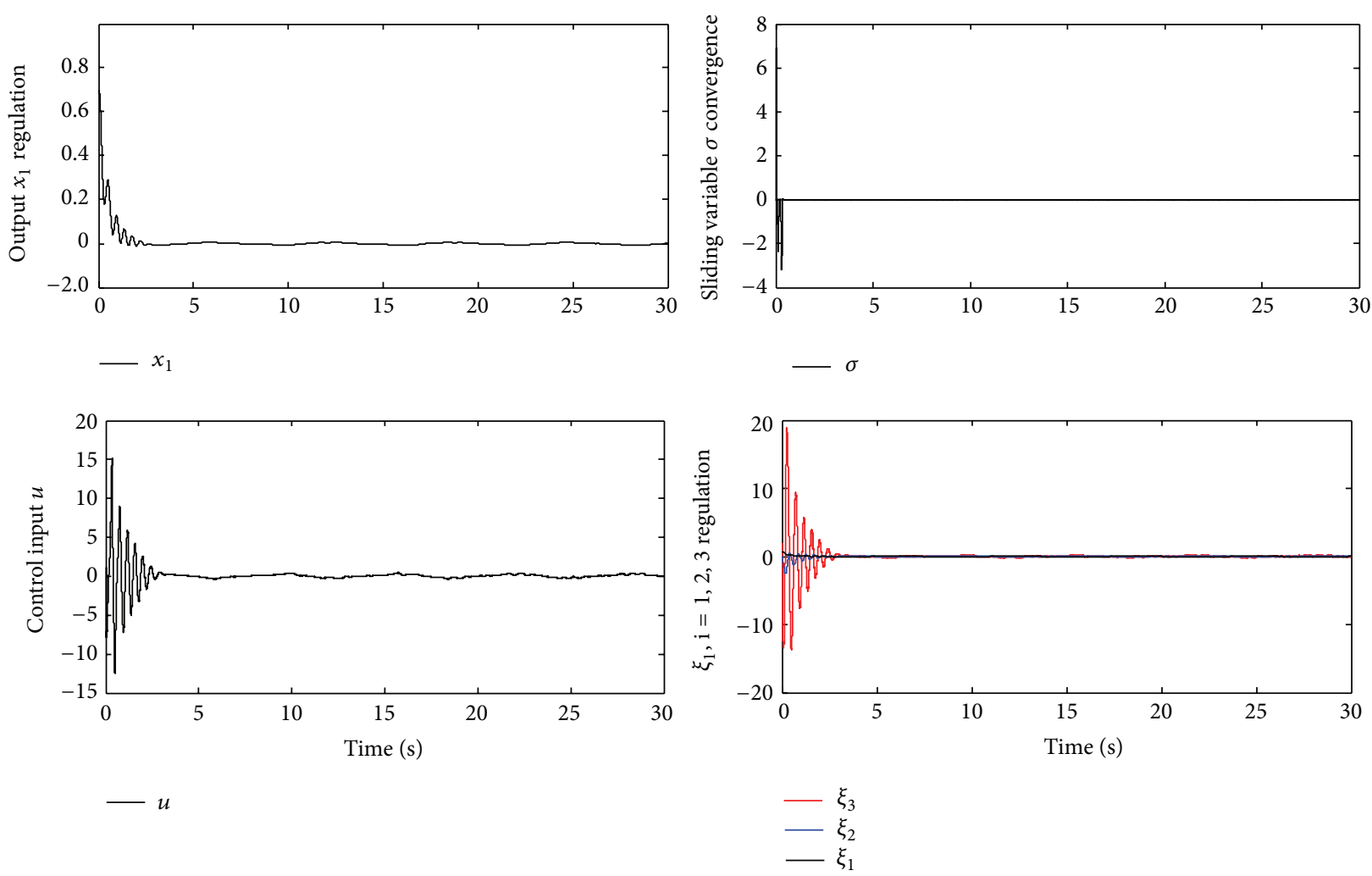

FIGURE 3: Output regulation, control effort, sliding variable convergence, and $\left[\xi_{1}, \xi_{2}, \xi_{3}\right]^{T}$ regulation in the presence of matched and unmatched uncertainty via the proposed control law.

TABLE 1: The parameters values of the control law used in the simulations.

\begin{tabular}{lcccccc}
\hline Constants & $k_{1}$ & $k_{2}$ & $k_{3}$ & $c_{1}$ & $c_{2}$ & $c_{3}$ \\
\hline 2-QCSMC & - & - & - & - & - & - \\
Proposed control law with $r=2$ & 490.2 & 180.7 & 5.9 & 6 & 5 & - \\
\hline
\end{tabular}

$f_{i}(x, t)=0$ for $\left.i=1,2,3\right)$ is simulated to confirm the aforementioned claim of the compensation of uncertain terms. This test with matched uncertainty is also performed with 2-QCSMC previously mentioned. The results are reported in Figures 1 and 2. In these Figures, it can be seen that the output system with state vector $\left[\xi_{1}, \xi_{2}, \xi_{3}\right]^{T}$ is regulated in the presence of uncertainties. It is noticeable that the proposed methodology provides a satisfactory regulation of the system output via a continuous control law. The 2-QCSMC also provides excellent performance yet with a control law which becomes discontinuous when the output regulation objective is attained. Apart from that, both the controllers need to use a differentiator $[16,18]$ to construct the derivatives of the output variable necessary in the control laws.

Case 2 (system operated under matched and unmatched uncertainties). In this section, the test with both matched and unmatched uncertainty is performed. The results with the proposed control law are depicted in Figure 3. These simulation results confirm the robust and chattering free nature of the proposed controller as well as its capability of efficiently solving the regulation problem even in this particularly critical case. In view of the nature of the uncertainty now considered, we cannot compare our results with those of the 2QCSMC algorithm, since that algorithm was designed under the assumption of having only matched uncertainty [18].

Note that the controller gains and the controllers parameters in both the experiments are listed in Table 1.

\section{Conclusions}

In this work, an output feedback dynamic sliding mode controller is presented which is capable of dealing with a class of SISO nonlinear systems operating under both matched and unmatched state dependent uncertainties. The uncertain system output trajectories are asymptotically regulated to zero inspite of the presence of the uncertainties, while a sliding mode is enforced in finite time along an integral manifold. The use of the integral sliding manifold allows one to subdivide the control design procedure into two steps. First a linear control component is designed by pole placement and then a discontinuous control component is added so as to cope with the uncertainty presence. The design procedure is relying on a suitably transformed system which 
generally appears in a canonical form and the control input appears with $k$ time derivatives. As a consequence, the control acting on the original system is obtained as the output of a chain of integrators and is, accordingly, continuous. This can be a clear benefit in many applications, such as those of mechanical nature, where a discontinuous control action could be nonappropriate or even disruptive for the actuators and system's health.

\section{Conflict of Interests}

The authors declare that there is no conflict of interests regarding the publication of this paper.

\section{References}

[1] C. Edwards and S. K. Spurgeon, Sliding Modes Control: Theory and Applications, Taylor \& Francis, London, UK, 1998.

[2] M. Fliess, "Generalized controller canonical forms for linear and nonlinear dynamics," IEEE Transactions on Automatic Control, vol. 35, no. 9, pp. 994-1001, 1990.

[3] H. S. Ramirez, "On the dynamical sliding mode control of nonlinear systems," International Journal of Control, vol. 57, no. 5, pp. 1039-1061, 1993.

[4] X.-Y. Lu and S. K. Spurgeon, "Output feedback stabilization of SISO nonlinear systems via dynamic sliding modes," International Journal of Control, vol. 70, no. 5, pp. 735-759, 1998.

[5] V. I. Utkin, Sliding Modes in Control Optimization, Springer, Berlin, Germany, 1992.

[6] J. C. Scarratt, A. Zinober, R. E. Mills, M. Rios-Bolívar, A. Ferrara, and L. Giacomini, "Dynamical adaptive first and secondorder sliding backstepping control of nonlinear nontriangular uncertain systems," Journal of Dynamic Systems, Measurement and Control, vol. 122, no. 4, pp. 746-752, 2000.

[7] D. Swaroop, J. K. Hedrick, P. P. Yip, and J. C. Gerdes, "Dynamic surface control for a class of nonlinear systems," IEEE Transactions on Automatic Control, vol. 45, no. 10, pp. 1893-1899, 2000.

[8] A. Ferrara and L. Giacomini, "On modular backstepping design with second order sliding modes," Automatica, vol. 37, no. 1, pp. 129-135, 2001.

[9] S. Tong and H.-X. Li, "Fuzzy adaptive sliding-mode control for MIMO nonlinear systems," IEEE Transactions on Fuzzy Systems, vol. 11, no. 3, pp. 354-360, 2003.

[10] S.-C. Tong, X.-L. He, and H.-G. Zhang, "A combined backstepping and small-gain approach to robust adaptive fuzzy output feedback control,' IEEE Transactions on Fuzzy Systems, vol. 17, no. 5, pp. 1059-1069, 2009.

[11] S. Tong, C. Liu, and Y. Li, "Fuzzy-adaptive decentralized outputfeedback control for large-scale nonlinear systems with dynamical uncertainties," IEEE Transactions on Fuzzy Systems, vol. 18, no. 5, pp. 845-861, 2010.

[12] V. I. Utkin, J. Guldner, and J. Shi, Sliding Mode Control in Electromechanical Systems, Taylor \& Francis, London, UK, 1999.

[13] A. Levant and L. Alelishvili, "Integral high-order sliding modes," IEEE Transactions on Automatic Control, vol. 52, no. 7, pp. 1278-1282, 2007.

[14] G. Bartolini, A. Ferrara, and E. Usai, "Output tracking control of uncertain nonlinear second-order systems," Automatica, vol. 33, no. 12, pp. 2203-2212, 1997.
[15] G. Bartolini, A. Ferrara, and E. Usai, "Chattering avoidance by second-order sliding mode control," IEEE Transactions on Automatic Control, vol. 43, no. 2, pp. 241-246, 1998.

[16] A. Levant, "Higher-order sliding modes, differentiation and output-feedback control," International Journal of Control, vol. 76, no. 9-10, pp. 924-941, 2003.

[17] I. Boiko, L. Fridman, and M. I. Castellanos, "Analysis of secondorder sliding-mode algorithms in the frequency domain," IEEE Transactions on Automatic Control, vol. 49, no. 6, pp. 946-950, 2004.

[18] A. Levant, "Quasi-continuous high-order sliding mode controllers," IEEE Transaction on Automatic Control, vol. 50, no. 11, pp. 1812-1816, 2005.

[19] F. Dinuzzo and A. Ferrara, "Higher order sliding mode controllers with optimal reaching," IEEE Transactions on Automatic Control, vol. 54, no. 9, pp. 2126-2136, 2009.

[20] H. H. Choi, "Variable structure output feedback control design for a class of uncertain dynamic systems," Automatica, vol. 38, no. 2, pp. 335-341, 2002.

[21] P. Park, D. J. Choi, and S. G. Kong, "Output feedback variable structure control for linear systems with uncertainties and disturbances," Automatica, vol. 43, no. 1, pp. 72-79, 2007.

[22] J. Xiang, W. Wei, and H. Su, "An ILMI approach to robust static output feedback sliding mode control," International Journal of Control, vol. 79, no. 8, pp. 959-967, 2006.

[23] J. M. A. da Silva, C. Edwards, and S. K. Spurgeon, "Slidingmode output-feedback control based on LMIs for plants with mismatched uncertainties," IEEE Transactions on Industrial Electronics, vol. 56, no. 9, pp. 3675-3683, 2009.

[24] W.-J. Cao and J.-X. Xu, "Nonlinear integral-type sliding surface for both matched and unmatched uncertain systems," IEEE Transactions on Automatic Control, vol. 49, no. 8, pp. 1355-1360, 2004.

[25] F. Castaños and L. Fridman, "Analysis and design of integral sliding manifolds for systems with unmatched perturbations," IEEE Transactions on Automatic Control, vol. 51, no. 5, pp. 853858, 2006.

[26] M. Rubagotti, A. Estrada, F. Castaños, A. Ferrara, and L. Fridman, "Integral sliding mode control for nonlinear systems with matched and unmatched perturbations," IEEE Transactions on Automatic Control, vol. 56, no. 11, pp. 2699-2704, 2011.

[27] J.-L. Chang, "Dynamic output integral sliding-mode control with disturbance attenuation," IEEE Transactions on Automatic Control, vol. 54, no. 11, pp. 2653-2658, 2009.

[28] Q. Khan, A. I. Bhatti, and Q. Ahmed, "Dynamic integral sliding mode control of nonlinear SISO systems with states dependent matched and mismatched uncertainties," in Proceedings of the 18th World Congress of the International Federation of Automatic Control (IFAC '11), pp. 3932-3937, Milan, Italy, September 2011.

[29] A. Isidori, Nonlinear Control Systems, Springer, 3rd edition, 1995.

[30] M. Fliess, "Nonlinear control theory and differential algebra," in Modeling and Adaptive Control, C. Byrness and A. Kurzhanski, Eds., vol. 105 of Lecture Notes in Control and Information Science, pp. 134-145, Springer, New York, NY, USA, 1988.

[31] Q. Khan, A. I. Bhatti, S. Iqbal, and M. Iqbal, "Dynamic integral sliding mode for MIMO uncertain nonlinear systems," International Journal of Control, Automation and Systems, vol. 9, no. 1, pp. 151-160, 2011.

[32] G. Bartolini, A. Pisano, E. Punta, and E. Usai, "A survey of applications of second-order sliding mode control to mechanical systems," International Journal of Control, vol. 76, no. 9-10, pp. 875-892, 2003. 

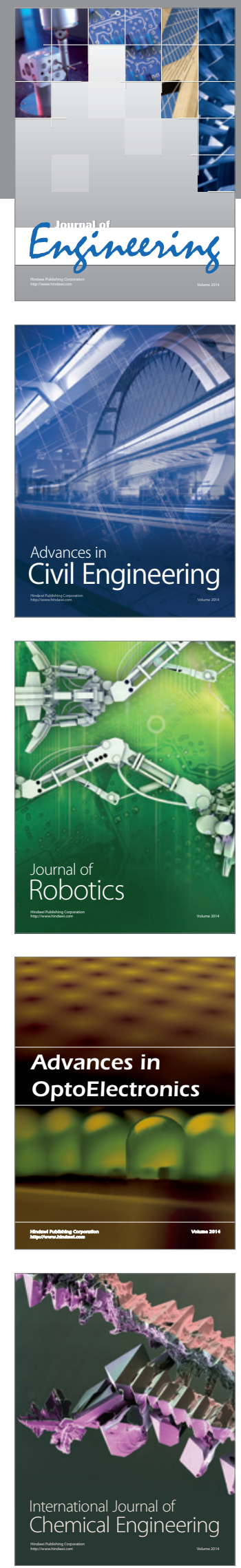

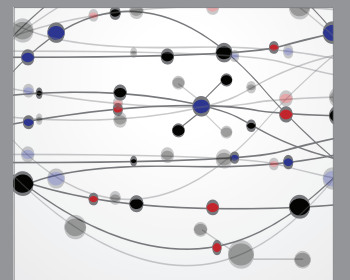

The Scientific World Journal
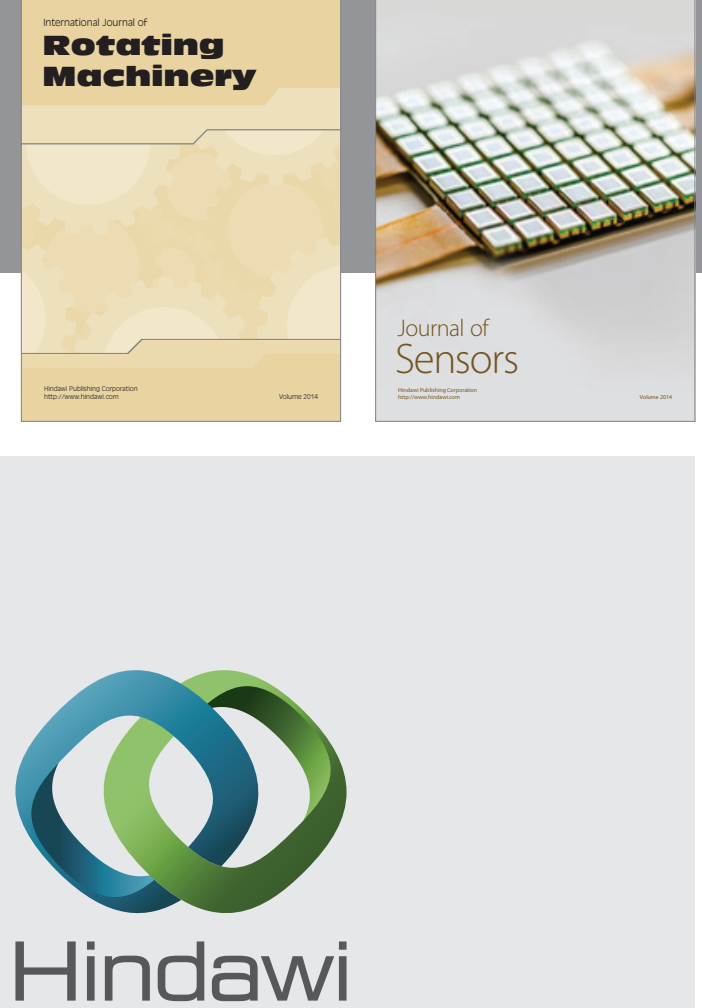

Submit your manuscripts at http://www.hindawi.com
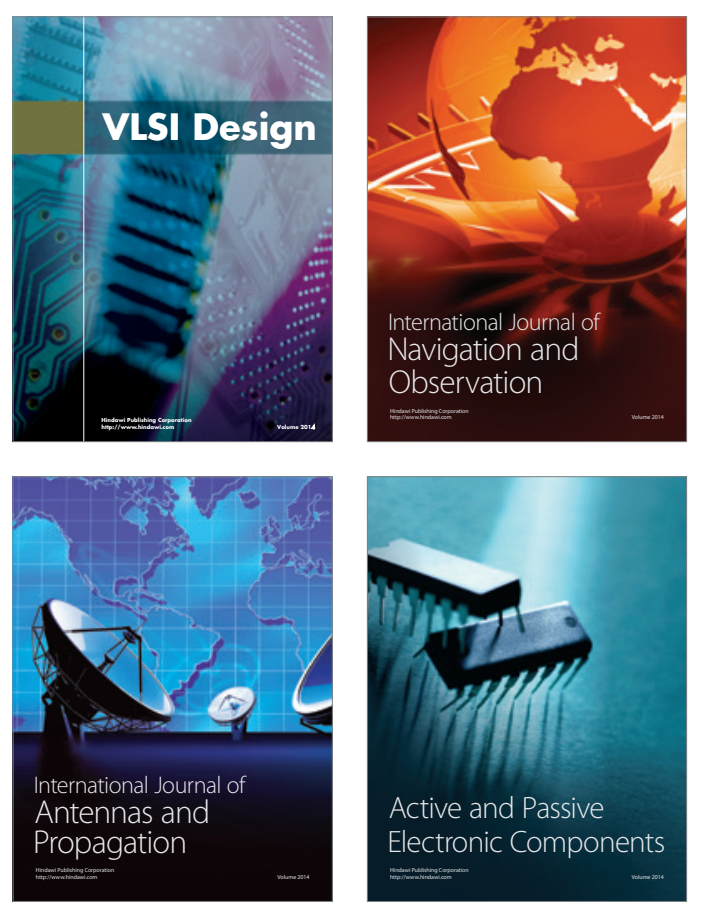
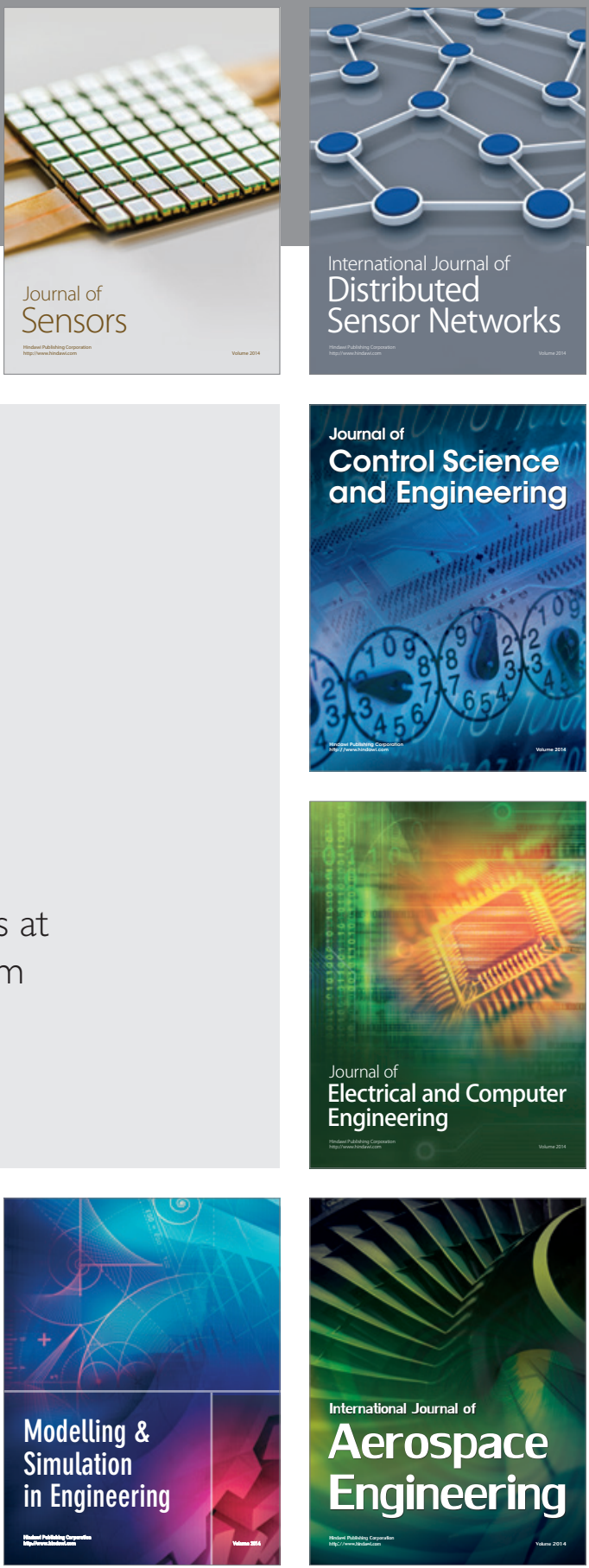

Journal of

Control Science

and Engineering
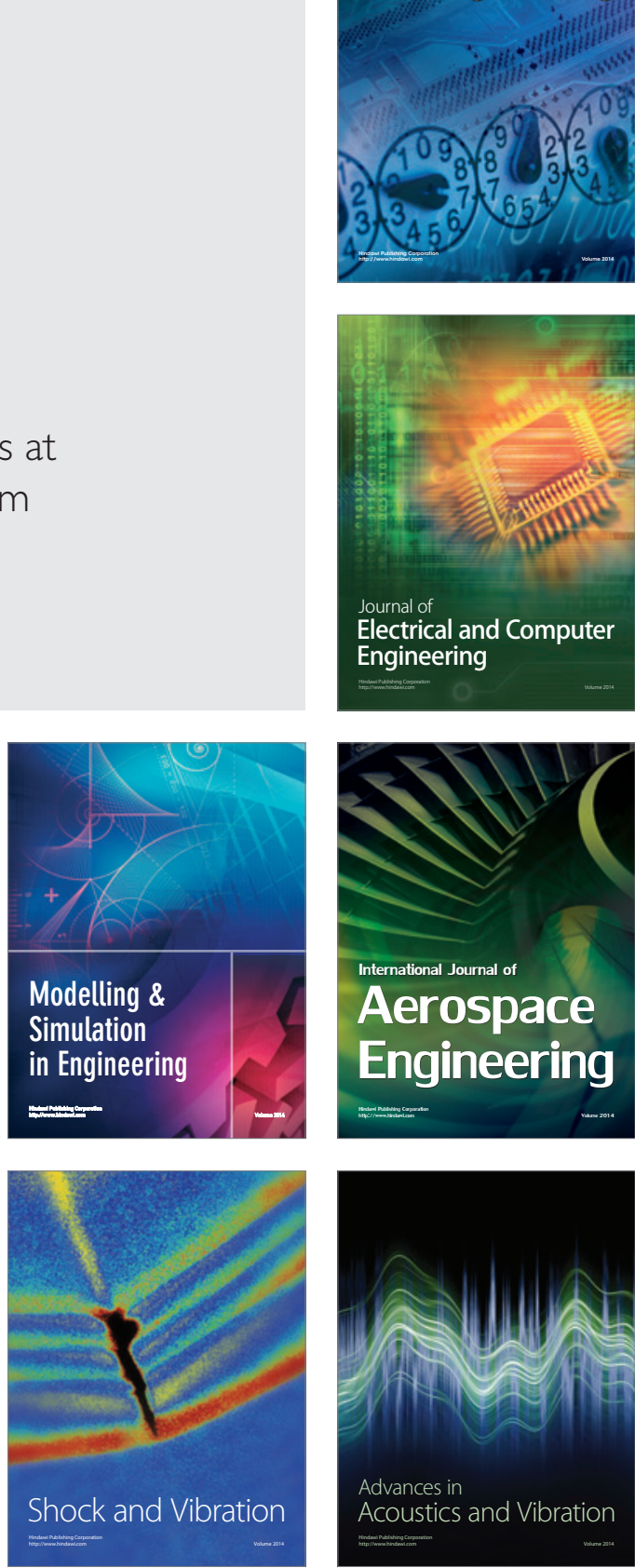